\title{
Hemolyzed blood as a clue to the diagnosis of abdominal pain
}

\author{
Raseen Tariq $^{1} \cdot$ Sahil Khanna ${ }^{1}$
}

Received: 12 August 2015/Accepted: 21 August 2015/Published online: 7 September 2015

(C) SIMI 2015

\section{Clinical case}

A 49-year-old man presented with nausea and vomiting, along with epigastric and periumbilical abdominal pain for 2 days. He denied hematemesis, diarrhea or hematochezia. Pain was constant, and was rated severe and radiated to his back. Past medical history was notable for obesity and hypertension. He did not smoke, but consumed four alcoholic drinks per week. Vital signs were notable for tachycardia. The physical examination revealed epigastric tenderness with no rebound. Initial laboratory evaluation showed a leukocytosis of 15,300 cells/ $\mu \mathrm{L}$. However, an accurate hemoglobin, hematocrit and serum chemistries (electrolytes, renal function tests and liver enzymes) were unobtainable due to repeatedly hemolyzed blood samples in the laboratory (Fig. 1a).

A glimpse of the blood samples from the laboratory showed milky white serum. Serum lipid analysis showed a triglyceride level of $4400 \mathrm{mg} / \mathrm{dL}$ (normal $<150 \mathrm{mg} / \mathrm{dL}$ ), and an LDL of $760 \mathrm{mg} / \mathrm{dL}$ (normal $<130 \mathrm{mg} / \mathrm{dL}$ ). This was the likely cause of the hemolyzed blood samples. Abdominal CT scan showed mild pancreatic enlargement of the body and tail of the pancreas, with peri-pancreatic inflammatory changes suggestive of acute pancreatitis (Fig. 1b).

Clinically, there were no signs of hyperviscosity syndrome. He was diagnosed with pancreatitis secondary to

Sahil Khanna

khanna.sahil@mayo.edu

1 Division of Gastroenterology and Hepatology, Mayo Clinic, 200 First Street SW, Rochester, MN 55905, USA hypertriglyceridemia, and was managed with aggressive intravenous hydration with dextrose in normal saline along with intravenous insulin, oral omega- 3 fatty acids, fenofibrate and atorvastatin to decrease his triglyceride levels. His abdominal pain improved, LDL improved to $86 \mathrm{mg} / \mathrm{dL}$ and triglycerides to $1480 \mathrm{mg} / \mathrm{dL}$ in $24 \mathrm{~h}$ and $563 \mathrm{mg} / \mathrm{dL}$ in $48 \mathrm{~h}$. Subsequent blood samples were not hemolyzed (Fig. 1a). He tolerated oral intake, and was discharged home on fenofibrate, atorvastatin, omega- 3 fatty acids, a low fat, low cholesterol diet and alcohol abstinence.

Severe hypertriglyceridemia ( $>1000 \mathrm{mg} / \mathrm{dL}$ ) may cause acute pancreatitis, but the mechanism remains unknown. Patients with severe hypertriglyceridemia should be evaluated for disorders of lipoprotein metabolism [1]. Pancreatitis secondary to hypertriglyceridemia is usually seen along with diabetes mellitus, diabetic ketoacidosis, hypothyroidism, increased alcohol intake or hormone replacement. Hemolysis is a rare complication of hyperlipidemia, and likely occurs due to destabilization of red cell membranes in the presence of lipemia [2]. In our patient, the repeatedly hemolyzed blood samples caused the inability to detect electrolyte abnormalities or elevations in amylase and lipase. Patients presenting with abdominal pain and repeatedly hemolyzed blood samples should lead one to pursue a diagnosis of severe hypertriglyceridemia induced pancreatitis. Other potential etiologies of hemolysis (intravascular and extravascular) include: defects of glucose-6-phosphate deficiency, hemoglobinopathies, autoimmune hemolytic anemia, microangiopathies, infections (such as malaria), pregnancy complications such as abruption with mild trauma, liver disease, hypersplenism, exposure to oxidants (e.g. aniline dyes), as well as snake and spider bites.

Treatment for hypertriglyceridemia-induced pancreatitis is intravenous insulin as it leads to increased lipoprotein 
Fig. 1 a Hemolyzed blood samples (at presentation) on the left and follow-up blood samples (after 3 days) with no hemolysis. b A representative CT scan image of acutely inflamed pancreas with heterogeneity and stranding
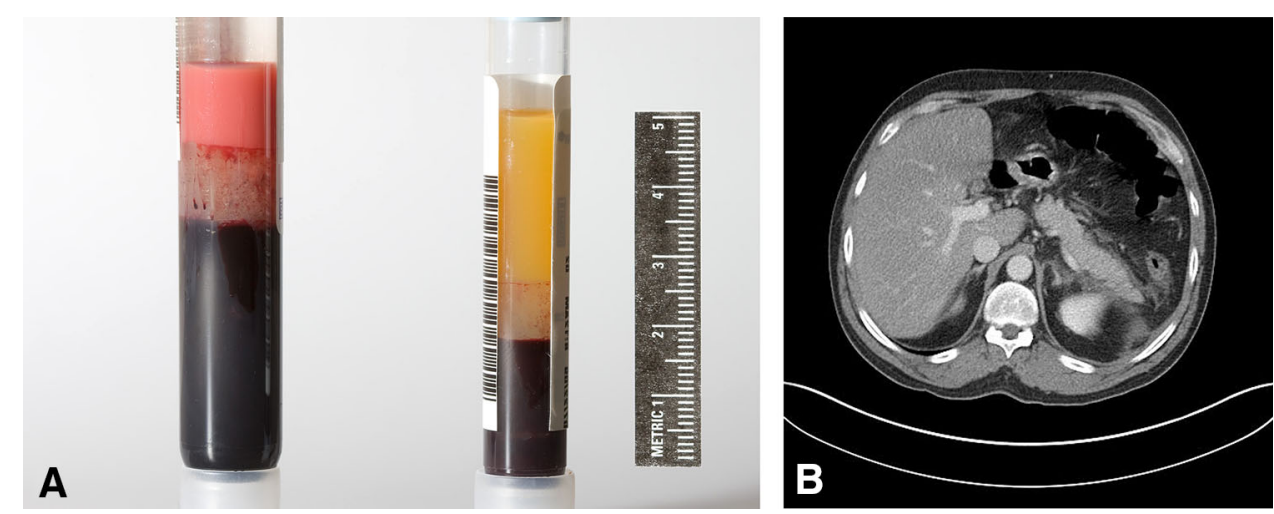

lipase production, and a decrease in triglyceride levels. In most cases, maintaining the TG level less than $500 \mathrm{mg} / \mathrm{dL}$ has been associated with marked clinical improvement [1]. In addition, patients with severe hypertriglyceridemia usually have a concomitant history of diabetes mellitus and the presence of hyperglycemia along with hypertriglyceridemia. Our patient had normal blood glucose levels, and was hence managed with dextrose infusion along with insulin. In patients with hyperglycemia associated with hypertriglyceridemia, intravenous insulin alone is given. Alternate treatment options include intravenous heparin and plasmapheresis [3]. The use of intravenous heparin alone has been under debate as it does helps initially in increasing lipoproteins; however, its long-term use has been linked with lipoprotein lipase deficiency [1]. Plasmapheresis is usually reserved for patients exhibiting hyperviscosity syndrome, or when other measures fail. Once the initial episode is treated, anti-hyperlipidemics should be initiated. For isolated hypertriglyceridemia, fibrates have been found to most effective as compared to niacin and statins [1].
Compliance with ethical standards

Conflict of interest The authors declare that they have no conflict of interest.

Statement of human and animal rights All procedures performed in studies involving human participants were in accordance with the ethical standards of the institutional and/or national research committee and with the 1964 Helsinki declaration and its later amendments or comparable ethical standards.

Informed consent Informed consent was obtained from all individual participants included in the study.

\section{References}

1. Tsuang W, Navaneethan U, Ruiz L et al (2009) Hypertriglyceridemic pancreatitis: presentation and management. Am J Gastroenterol 104(4):984-991

2. Dimeski G, Mollee P, Carter A (2005) Increased lipid concentration is associated with increased hemolysis. Clin Chem 51(12):2425

3. Cole RP (2009) Heparin treatment for severe hypertriglyceridemia in diabetic ketoacidosis. Arch Intern Med 169(15):1439-1441 